\title{
Li-ion Battery Research in Hierarchical Green-Energy Materials Research Center ${ }^{\dagger}$
}

\author{
Jow-Lay Huang ${ }^{1,2 *}$, Alex Chinghuan Lee ${ }^{2}$ \\ 1 Department of Materials Science and Engineering, National Cheng Kung University, Tainan, Taiwan, R.O.C. \\ 2 Hierarchical Green-Energy Research Center, National Cheng Kung University, Tainan, Taiwan, R.O.C. \\ * Correspondence: jlh888@ mail.ncku.edu.tw; \\ $\dagger$ Presented at Materials Chemistry and Physics (Materials Chemistry 2020) - International e-Conference
}

Received: 16.09.2020; Revised: 20.09.2020; Accepted: 24.09.2020; Published: 27.09.2020

\begin{abstract}
Hierarchical Green-Energy Research Center aims to promote materials innovation of Li-ion battery (LIB) for electric scooter and MWh class energy storage. Our team has mainly developed strategies for material optimization and research methodology for the improvement of electric power and cyclic stability of LIB. Metal oxides are abundant in defect structures that determine electric conductance and electrochemical activity. The first principle calculation of lithium titanate (LTO) was applied to access the electronic structure of pristine LTO and aliovalent ion-doping LTO. The other oxides were chosen as nanostructured anode materials anchored on reduced graphene oxide. We found that their cyclic ability is significantly improved due to the structurally synergetic effect between oxide nanocrystallite and substrate; $\mathrm{Si}$ is regarded as one of the most popular anode materials for 3rd generation LIB, but its cycle life is still limited by an overgrowth of secondary electrolyte interface (SEI). Glucose-derived Si-O-C ligand in modified SEI possessed a high affinity to Li-chelation, thus alleviating volume expansion and structural instability. Electrolyte design is considered the current technical bottleneck for the implementation of high voltage LIB. An ionic liquid hybrid electrolyte has been demonstrated excellent oxidation potential of the carbonate solvent, resistance to $\mathrm{Al}$ corrosion, and potential of $\sim 5.0 \mathrm{~V}$ (vs. $\mathrm{Li}+/ \mathrm{Li}$ ) even at an operating temperature of $55 \mathrm{oC}$. Another highlight in the center is gel polymer electrolyte (GPE) that enables enhancement of lithium-ion transference number of 0.57 by the existence of the imidazolium group. The newly designed GPE guaranteed effective Li+ pathways in electrolyte bulk and at electrode/electrolyte interfaces, a high potential of $5.4 \mathrm{~V}$, and simplicity of electrolyte synthesis and all-solid-state battery assembly for industrial-scale production processes.
\end{abstract}

Keywords: Li-ion battery; intercalation; lithium titanate; Si anode; ionic liquid; gel polymer electrolyte; secondary electrolyte interface.

(C) 2020 by the authors. This article is an open-access article distributed under the terms and conditions of the Creative Commons Attribution (CC BY) license (https://creativecommons.org/licenses/by/4.0/).

\section{Funding}

This research was funded by the Hierarchical Green-Energy Materials (Hi-GEM) Research Center, from The Featured Areas Research Center Program within the framework of the Higher Education Sprout Project by the Ministry of Education (MOE) and the Ministry of Science and Technology (MOST 109-2634-F-006 -020) in Taiwan.

\section{Acknowledgments}

This research has no acknowledgment. 


\section{Conflicts of Interest}

The authors declare no conflict of interest. 